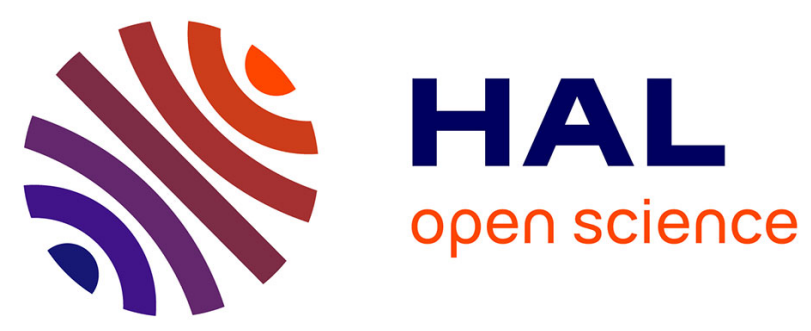

\title{
How to Flip a Bit?
}

Michel Agoyan, Jean-Max Dutertre, Amir-Pasha Mirbaha, David Naccache, Anne-Lise Ribotta, Assia Tria

\section{To cite this version:}

Michel Agoyan, Jean-Max Dutertre, Amir-Pasha Mirbaha, David Naccache, Anne-Lise Ribotta, et al.. How to Flip a Bit?. On-Line Testing Symposium (IOLTS), 2010 IEEE 16th International, Jul 2010, Corfu, Greece. 10.1109/IOLTS.2010.5560194 . emse-01130826

\section{HAL Id: emse-01130826 https://hal-emse.ccsd.cnrs.fr/emse-01130826}

Submitted on 12 Mar 2015

HAL is a multi-disciplinary open access archive for the deposit and dissemination of scientific research documents, whether they are published or not. The documents may come from teaching and research institutions in France or abroad, or from public or private research centers.
L'archive ouverte pluridisciplinaire HAL, est destinée au dépôt et à la diffusion de documents scientifiques de niveau recherche, publiés ou non, émanant des établissements d'enseignement et de recherche français ou étrangers, des laboratoires publics ou privés. 


\title{
How to Flip a Bit?
}

\author{
Michel Agoyan*, Jean-Max Dutertre ${ }^{\dagger}$, Amir-Pasha Mirbaha ${ }^{\dagger}$, David Naccache ${ }^{\ddagger}$, Anne-Lise Ribotta ${ }^{\dagger}$ and Assia Tria* \\ ${ }^{*}$ Département Systèmes et Architectures Sécurisées (SAS) \\ ${ }^{*}$ CEA-LETI, Gardanne, France \\ \{michel.agoyan, assia.tria\}ecea.fr \\ †École Nationale Supérieure des Mines de Saint-Étienne (ENSMSE), Gardanne, France \\ \{dutertre, mirbaha, ribotta\}eemse.fr \\ †Équipe de cryptographie, École normale supérieure, Paris, France \\ david.naccache@ens. fr
}

\begin{abstract}
This note describes laser fault experiments on an 8bit $0.35 \mu \mathrm{m}$ microcontroller with no countermeasures. We show that reproducible single-bit faults, often considered unfeasible, can be obtained by careful beam-size and shot-instant tuning.
\end{abstract}

\section{INTRODUCTION}

Fault attacks consist in using hardware malfunction to infer secrets from the target's faulty outputs. Within fault attacks, Differential Fault Analysis [2] (DFA) is a particular analysis technique exploiting differences between correct and faulty outputs. We refer the reader to [14] for more information on fault injection techniques.

This note describes laser faults experiments on an 8-bit $0.35 \mu \mathrm{m}$ RISC microcontroller with no countermeasures. We show that reproducible single-bit faults, often considered unfeasible, can be obtained by careful beam-size and shot-instant tuning. Moreover, we obtain such faults even when the beam's impact area exceeds a single SRAM cell. This underlines the need to protect small data objects, such as pointers, counters or flags, against "surgical" faults targeting a single-bit on a specific byte in memory and nothing else.

\section{THE AdVAnced EnCRYPtion StAndard}

We assume that the reader is familiar with the AES [10] that we recall here for the ease of reference.

The AES-128 (hereafter AES) encrypts 128-bit plaintexts into 128-bit ciphertexts using a 128-bit key $K$. The algorithm performs 10 rounds (after a short initial round) and consists of two separated processes: a key schedule that derives round keys and the encryption routine itself. During decryption key schedule is reversed and encryption is replaced by a very similar decryption process.

The initial round uses $K_{0}=K$ as a round key; for all subsequent rounds, new round keys $K_{i}$ are calculated from their predecessors $K_{i-1}$. Figure 1 illustrates the AES' structure.

In most implementations the $K_{i} \mathrm{~s}$ are computed and stored in memory before encryption starts. Encryption treats the 16byte plaintext $M$ as a $4 \times 4$ byte matrix. Each round, except the initial and the final, includes four steps: A substitution of the matrix's contents using a lookup table (SubBytes), a rotation

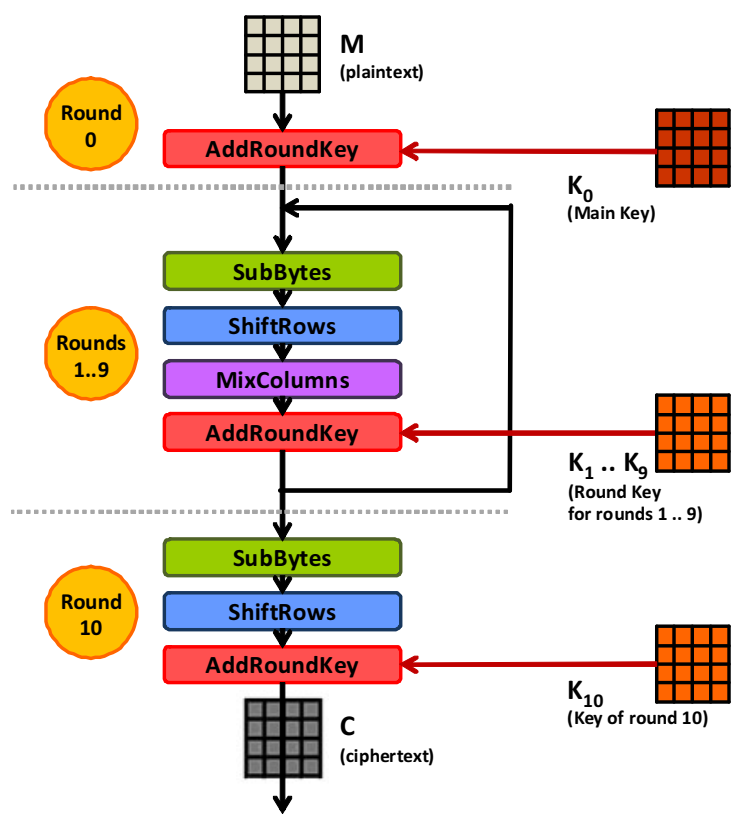

Fig. 1. The AES - General Outline.

of the matrix's rows (ShiftRows) and a linear transform in $\mathrm{GF}\left(2^{8}\right)$ (MixColumns) combining each matrix element with other column elements weighted by different coefficients $(1,2$ or 3$)$. At the end of each round, $K_{i}$ is xored with MixColumns's result (an operation called AddRoundKey).

\section{LASER FAULT INJECTION}

Laser (Light Amplification by Stimulated Emission of Radiation) is a stimulated-emission electromagnetic radiation in the visible or the invisible domain. Laser light is monochromatic, unidirectional, coherent and artificial (i.e. laser does not spontaneously exist in nature). Laser light can be generated as a beam of very small diameter (a few $\mu \mathrm{m}$ ). The beam can pass through various material obstacles before impacting a target during a very short duration.

Laser impacts on electronic circuits are known to alter functioning. Current chip manufacturing technologies are in 


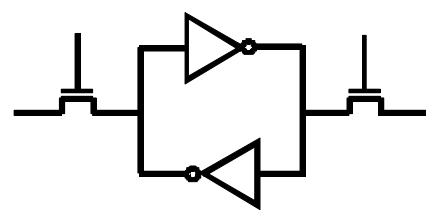

Fig. 2. Architecture of a typical SRAM cell.

the nanometers range. This, and the laser's brief and precise reaction time, makes laser a particularly suitable fault injection means.

\section{A. Photoelectrical Effects of Laser on Silicon}

SRAM (Static Random Access Memory) laser exposure is known to cause bit-flips [13], [6], [1], [5], a phenomenon called Single Event Upset (SEU). By tuning the beam's energy level below a destructive threshold, the target will not suffer any permanent damage.

A conventional one-bit SRAM cell (Figure 2) is made of two cross-coupled inverters. Every cell has two additional transistors controlling the cell's content access during write and read. As every inverter is made of two transistors, an SRAM cell contains six MOS.

In each cell, the states of four transistors encode the stored value. By design, the cell admits only two stable states: a " 0 " or a "1". In each stable state, two transistors are at an on state and two others are OFF.

If a laser beam hits the drain/bulk reversed-biased PN junctions of a blocked transistor, the beam's energy may create pairs of electrons as the beam passes through the silicon. The charge carriers induced in the collection volume of the drainsubstrate junction of the blocked transistor are collected and create a transient current that inverts logically the inverter's output voltage. This voltage inversion is in turn applied to the second inverter that switches to its opposite state: all in all, a bit flip happens [6], [1].

From the opponent's perspective, an additional advantage of laser fault injection is reproducibility. Identical faults can be repeated by carefully tuning the laser's parameters and the target's operating conditions.

\section{B. Different Parameters in a Fault Attack by Laser}

In a laser attack, the opponent usually controls the beam's diameter, wavelength, amount of emitted energy, impact coordinates (attacked circuit part) and the exposure's duration. Sometimes, the opponent may also control the impact's moment $^{1}$, the target's clock frequency, $V_{\mathrm{cc}}$ and temperature. Finally, laser attacks may indifferently target the chip's front side or back side.

However, the chip's front and back sides have different characteristics when exposed to a laser beam:

\footnotetext{
${ }^{1}$ i.e. the impact's synchrony with a given clock cycle of the target.
}

1) Front side attacks: are particularly suited to green wavelength $(\sim 532 \mathrm{~nm})$. The visibility of chips components makes positioning very easy in comparison to backside attacks. But because of the metallic interconnects' reflective effect, it is difficult to target a component with enough accuracy. In addition, progress in manufacturing technologies results in both a proliferation of metal interconnects and much smaller chips. All in all, it becomes increasingly difficult to hit a target area.

2) Backside attacks: are more successful at the infrared wavelength $(\sim 1064 \mathrm{~nm})$ as the laser needs to deeply enter the silicon. Positioning is more difficult for lack of visibility. Nonetheless, backside attacks allow to circumvent the reflective problem of metallic surfaces.

\section{GIRAUD's Bit DFA}

Differential Fault Analysis [2] (DFA) is an analysis technique exploiting differences between correct and faulty outputs. Several byte-level and bit-level AES DFA variants exist (e.g. [11], [8], [9], [7], [3]). Given the dependence of these attacks on precise "surgical" fault injection, the feasibility of bit/byte-level DFA remained somewhat unclear.

[8] describes a bit-level and a byte-level DFA on AES. The bit-level attack requires the injection of a single-bit fault into a specific byte of the temporary ciphertext result of the penultimate round $\left(M_{9}\right)$ (e.g. to inject such a fault into the 9-th round AddRoundKey or into the temporary ciphertext result just before the SubBytes input to the 10-th round).

To discover one byte of $K_{10}$, the attack requires to repeat a single-bit fault for at least three different plaintexts. The three faulty results are then compared to their corresponding correct ciphertexts to infer key information. We show that this attack can be implemented, even when the laser spot is wider than the SRAM's cell.

During normal processing, the value of each ciphertext $(C)$ cell is calculated by xoring a corresponding $K_{10}$ value with a temporary value resulting from the application of SubBytes (SB) and ShiftRows (SR) to $\left(M_{9}\right)$ :

$$
C=\mathrm{SR}\left[\mathrm{SB}\left(M_{9}\right)\right] \oplus K_{10}
$$

As shown in Figure 3, upon single-byte fault injection in $K_{9}$ (regardless the number of faulty bits) the faulty message will feature only one faulty byte that will leak information on one byte of $K_{10}$. Figure 4 shows the consequences of an injected fault in $K_{9}$ throughout the 9-th and 10-th rounds.

For the sake of clarity, we consider all subsequent equations bytewise thereby abstracting away ShiftRowss that do not affect individual byte values. Thus, (1) becomes (2):

$$
C=\mathrm{SB}\left(M_{9}\right) \oplus K_{10}
$$

By considering the injection of the single-bit fault $e$ on the 10 -th round SubBytes input, the faulty ciphertext $(D)$ can be expressed as (3): 


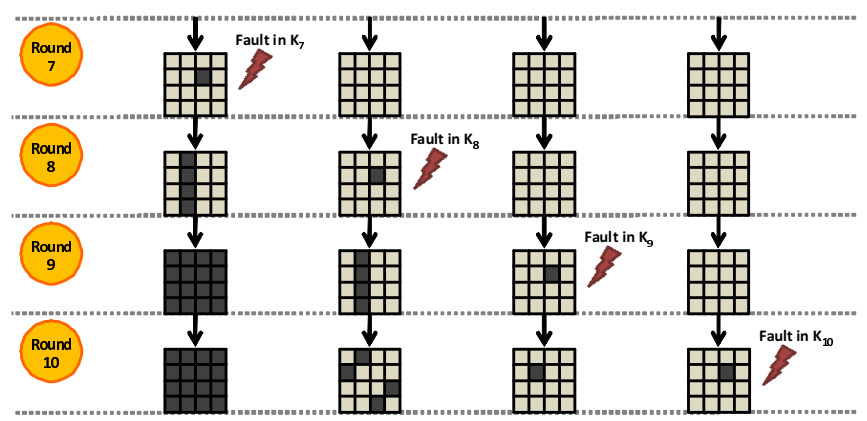

Fig. 3. Effects of one faulty round key byte at different rounds.

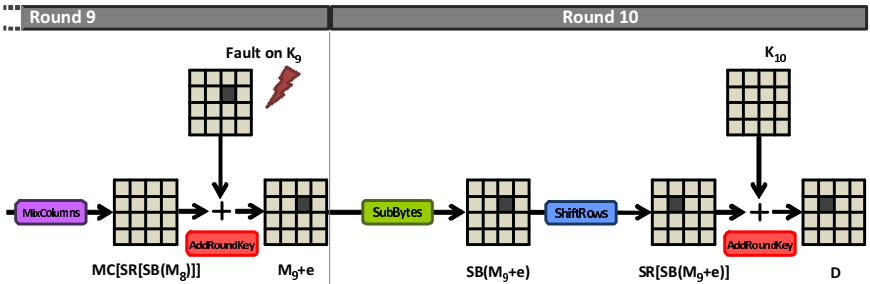

Fig. 4. Giraud's bit DFA.

$$
D=\operatorname{SB}\left(M_{9} \oplus e\right) \oplus K_{10}
$$

[8] observes that a xor between a faulty and a correct ciphertext reveals a difference $(\Delta=C \oplus D)$ corresponding to a set of hypotheses on the corresponding $M_{9}$ byte value before the attack, and on the injected single-bit fault $e$.

$$
\Delta=\mathrm{SB}\left(M_{9} \oplus e\right) \oplus \mathrm{SB}\left(M_{9}\right)
$$

(4) will yield a set of hypotheses on possible $M_{9}$ and $e$ value-pairs. Using (5), a corresponding $K_{10}$ value can be replaced for each pair of $\left(M_{9}, e\right)$ values.

$$
K_{10}=\mathrm{SB}\left(M_{9} \oplus e\right) \oplus D
$$

By repeating the fault injection for at least three different plaintexts, the opponent creates three sets of hypotheses on the corresponding $K_{10}$ byte value. Then, sets are intersected to spot the single hypothesis that reveals one $K_{10}$ byte. With a probability of about $97 \%$, three plaintexts suffice to discover a byte of $K_{10}$ [8]. Otherwise, the opponent iterates the process for more plaintexts to until the sets' intersection reaches a singleton. After finishing this operation for one byte of $K_{10}$, the procedure is repeated to discover $K_{10}$ 's remaining bytes. Finally, $K=K_{0}$ is inferred by reversing the key schedule.

\section{Practical Single-Bit Fault Injection}

Outline: After chip decapsulation and a mapping of the chip's components, we selected a large target area, given our knowledge of the implementation. Using automated search on the chip's front-side, we modified the impact's coordinates,
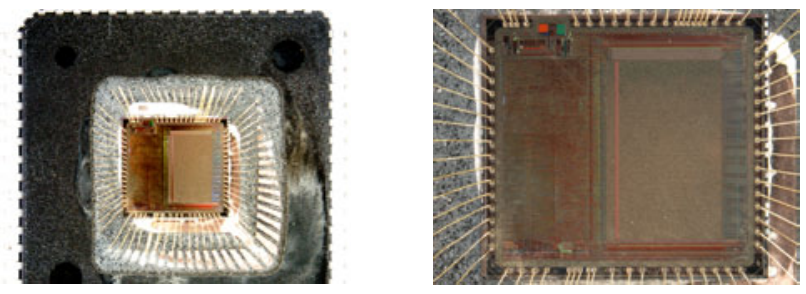

Fig. 5. Decapsulated chip (SRAm is on the left middle and bottom side).

the beam parameters and timing until a single bit fault was obtained. Finally, Giraud's bit DFA was performed.

The target is an 8-bit $0.35 \mu \mathrm{m} 16 \mathrm{MHz}$ RISC microcontroller with an integrated $4 \mathrm{~KB}$ SRAM and no countermeasures. The device runs SOSSE (Simple Operating System for Smartcard Education [4]) to which we added some commands, most notably for feeding-in cleartexts and retrieving ciphertexts. $K$ was embedded in the code. As encryption starts, the $K_{i}$ s are derived and stored in SRAM. The laser, shown in Figures 10 and 11 , is equipped with a YAG laser emitter in three different wavelengths: green, infrared and ultraviolet.

The spot's diameter can be set between 0 and $2500 \mu \mathrm{m}$. As the beam passes through a lens, it gets reduced by the lens' zoom factor and loses a big part of its energy. Our experiments were conducted with a $20 \times$ Mitutoyo lens, a green ${ }^{2}$ beam of $\varnothing 4 \mu \mathrm{m}$ and $\simeq 15 \mathrm{pJ}$ per shot $^{3}$. The circuit is installed on a programmable Prior Scientific $\mathrm{X}-\mathrm{Y}$ positioning table ${ }^{4}$. The X-Y table, card reader, laser and an FPGA trigger board, were connected via RS-232 to a control PC. The FPGA trigger board receives an activation signal from the reader and sends a trigger signal to the laser after a delay defined by the control PC.

Experiments were conducted in ambient temperature and at $V_{\mathrm{cc}}=5 \mathrm{~V}$. These parameters are within the device's normal operating conditions $2.7 \mathrm{~V} \leq V_{\mathrm{cc}} \leq 5.5 \mathrm{~V}$.

The chip was decapsulated by chemical etching using a Nisene JetEtch automated acid decapsulator. The decapsulator can be programmed for the chemical opening of different chip types using different ratios of nitric acid $\left(\mathrm{HNO}_{3}\right)$ and sulfuric acid $\left(\mathrm{H}_{2} \mathrm{SO}_{4}\right)$, at a desired temperature and during a specified time. For opening our chip, we used only nitric acid at $80^{\circ} \mathrm{C}$ for 40 seconds. The etched chip (Figure 5) successfully passed functional tests before and during fault injection.

As it is very difficult to target the chip's (ALU) (Arithmetic Logic Unit) during a very specific instant between the end of 9-th round and the beginning of the 10-th round, we decided to target $K_{9}$.

Finding the SRAM area containing $K_{9}$ and properly tuning the laser's parameters is very time consuming. The number of faults in $C$, their position and their contents indicate which round key has been hit. MixColumns will amplify any singlebit/byte fault occurring in any $K_{i}$ preceding $K_{9}$ and result in

\footnotetext{
$2532 \mathrm{~nm}$ wavelength.

${ }^{3}$ At the laser source emitter, before passing through the lens.

${ }^{4}$ Motorized stepper stage for upright microscopes with $0,1 \mu \mathrm{m}$ steps.
} 


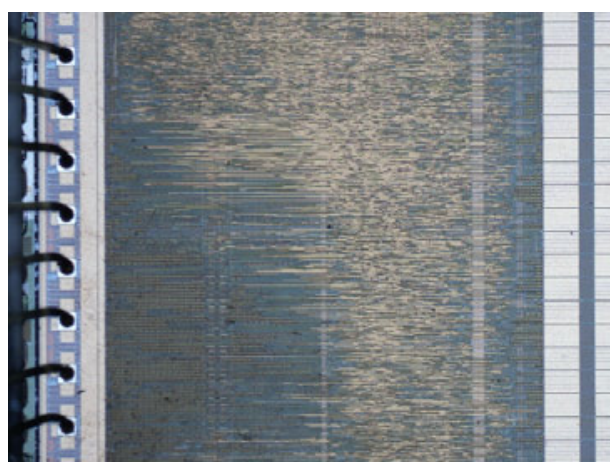

Fig. 6. Decapsulated chip (closeup on SRAM).
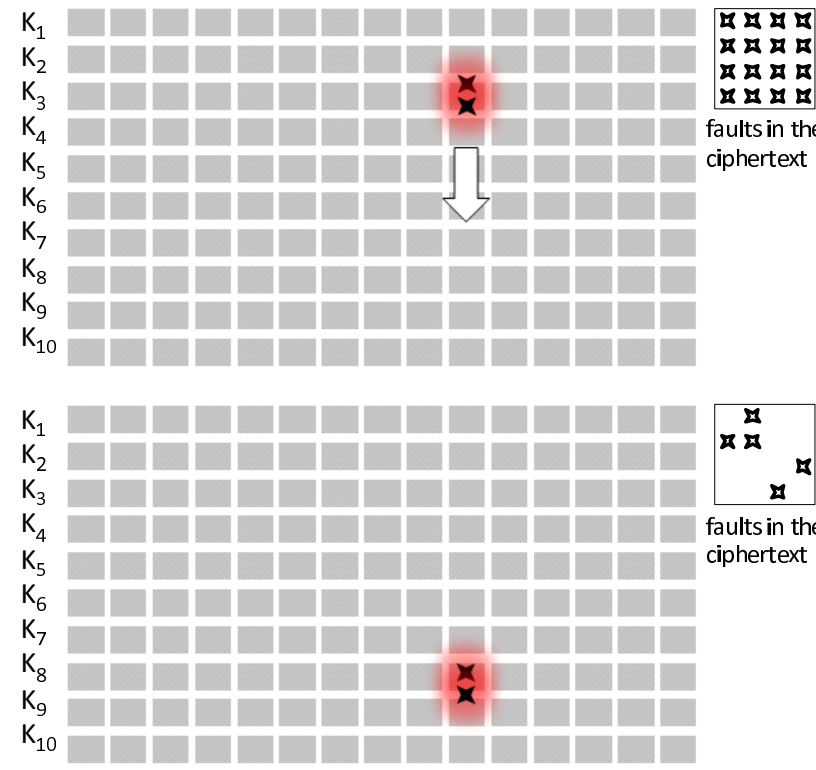

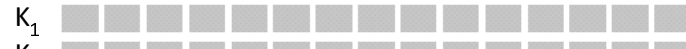

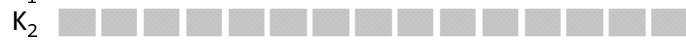

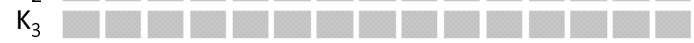
$\mathrm{K}_{4}+\mathrm{C}_{3}$ $\mathrm{K}_{5}$

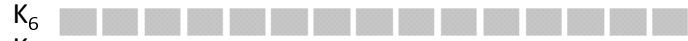
$\mathrm{K}_{7}+\mathrm{W}=\mathrm{W}$ $K_{8}$
$K^{2}$ $\mathrm{K}_{9}+\mathbf{K}=\mathbf{x}$ $x^{x} x$ faults in the ciphertext

$\mathrm{K}_{10} \mathrm{~W}=\mathrm{W}=\mathrm{a}$

Fig. 7. Exploration process.

a multi-byte or a fully faulty ciphertext (we call such a bad event an "early fault"). As shown in Figure 3, a single-bit/byte fault on $K_{8}$ changes 4 bytes and on any previous $K$ results in a completely faulty ciphertext while a fault in $K_{9}$ or $K_{10}$ changes only one byte. However, injected faults are not always limited to a single byte and/or to a single $K_{i}$. When more than 3 ciphertext bytes are faulty, it is difficult to determine if the observed result is due to an early fault or to several faults in $K_{9}$ and $K_{10}$ (Table I).

Figure 8 compares a $1 \mu \mathrm{m}$ laser spot and SRAM cells in

\begin{tabular}{|c|c|c|}
\hline & Transistor & SRAM Cell \\
\hline $350 \mathrm{~nm}$ & & \\
\hline $130 \mathrm{~nm}$ & 0 & \\
\hline $90 \mathrm{~nm}$ & 0 \\
\hline $65 \mathrm{~nm}$ & 0 & $\mathbf{3}$ \\
\hline
\end{tabular}

Fig. 8. $1 \mu \mathrm{m}$ laser spot (dotted circle) vs. technology sizes [12].

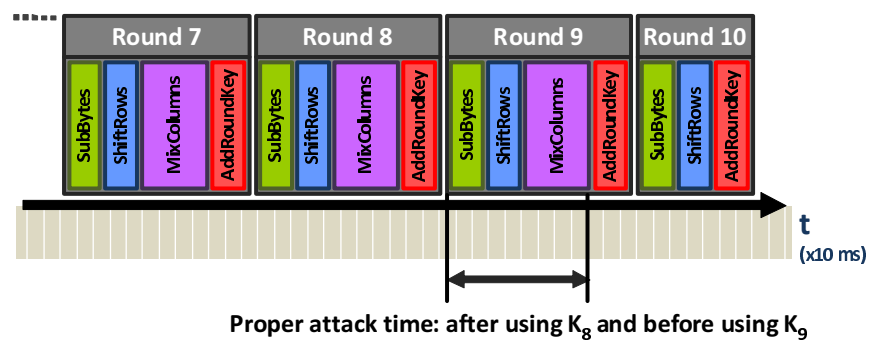

Fig. 9. Attack's timing.

TABLE I

Potential FAULTy $K_{i}$ S AS FUNCTION OF OBSERVED FAULTY CIPHERTEXT BYTES.

\begin{tabular}{|c|c|c|c|c|}
\hline number of faulty $C$ bytes & \multicolumn{5}{|c|}{ potential faulty round keys } \\
\hline & $K_{10}$ & $K_{9}$ & $K_{8}$ & previous round keys \\
\hline $1,2,3$ & $\checkmark$ & $\checkmark$ & & \\
\hline $4, \ldots, 15$ & $\checkmark$ & $\checkmark$ & $\checkmark$ & \\
\hline 16 & $\checkmark$ & $\checkmark$ & $\checkmark$ & $\checkmark$ \\
\hline
\end{tabular}

different technology sizes. As technology advances, transistor density per $\mu \mathrm{m}$ grows. With several transistors are packed into $1 \mu \mathrm{m}$ areas, single-bit fault injection will require much more precise equipment and are likely to become unfeasible using cheap lasers.

Despite fine-grained energy and spatial control we detected faults in keys neighboring $K_{9}$. To overcome this problem, we isolated $K_{10}$ from faults and restricted the shot to a $100 \mu \mathrm{s}$ interval between the use of $K_{8}$ and $K_{9}$ (Figure 9).

Figure 7 shows how we could confine faults to a singlebit of $K_{9}$. When physically more than one single-bit faulty byte existed, we could logically obtain a single-bit fault by controlling the laser's shooting time. Figure 7 is just a model of the real SRAM (Figure 6) to describe our technique and does not correspond to real address allocation. We could successfully inject a single-bit fault into each of the 16 bytes of $K_{9}$ and iterate the process for 4 different texts. This sufficed 

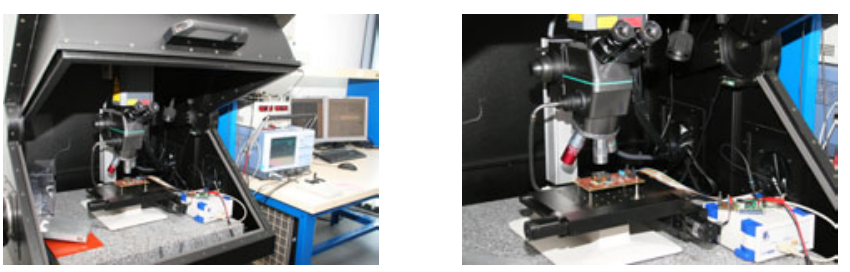

Fig. 10. Laser and target (general overview).

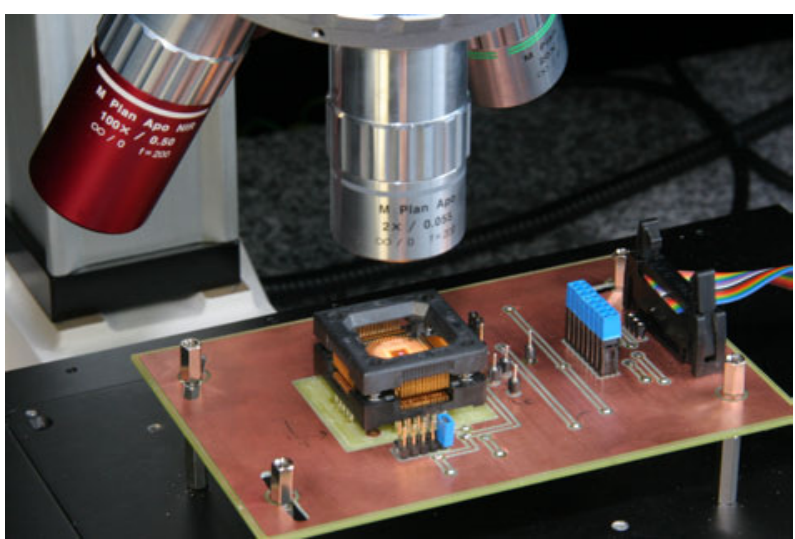

Fig. 11. Laser and target (closeup).

to succeed Giraud's bit DFA.

As shown in the topmost part of Figure 7, we searched $K_{9}$ 's precise storage area by monitoring the number and the type of faults in the ciphertext. Then (middle part of Figure 7), by a precise beam localization, we managed to inject faults only into $K_{9}$. This, however, did not turn out to be fully deterministic as sometimes we would also inflict faults to previous round keys. At that point (lowermost part of Figure 7), by fine-tuning spatial and temporal beam localization (just after the use of $K_{8}$ ), we managed to restrict the injected faults only to $K_{9}$. This is the exact assumption of Giraud's scenario for single-bit fault injection.

\section{CONCLUSION}

We implemented single-bit Giraud's attack [8] using laser fault injection. Whilst this is not the most effective fault attack on AES, this scenario is usually regarded as the most difficult as it requires to limit the attack to one single bit. This is much more stringent that most other AES fault attacks (e.g. [11], [9], [7], [3]) that target an entire byte, regardless the number of faulty bits. The experiments reported in this paper also apply to other attacks (e.g. [11], [9]) and underline the possibility to precisely modify even flags, counters, pointers and other single memory cells that control program flow, in the absence of countermeasures, even at a single-bit level.
In summary, this note's main conclusions are:

- It is possible to implement a single-bit laser fault attack on an AES round key.

- Even when it is physically impossible to target a singlebit on one byte because the beam hits a few other bytes, careful spatial and temporal coordination may allow to deceive the encryption process to consider logically only a single-bit fault that corresponds to Giraud's bit scheme.

- It is possible to reproduce the same faults on different plaintexts. This assesses the reality of bit-level Giraud's scenario on unprotected chips.

\section{REFERENCES}

[1] H. Bar-El, H. Choukri, D. Naccache, M. Tunstall and C. Whelan, The sorcerer's apprentice guide to fault attacks, Proceedings of the IEEE, vol. 94 (2), IEEE, 2006, pp. 370-382.

[2] E. Biham and A. Shamir, Differential fault analysis of secret key cryptosystems, Proceedings of Crypto'97, LNCS, vol. 1294, SpringerVerlag, 1997, pp. 513-525.

[3] J. Blömer and J.P. Seifert, Fault based cryptanalysis of the Advanced Encryption Standard (AES), Financial Cryptography - Proceedings of FC 2003, LNCS, vol. 2742, Springer-Verlag, 2003, pp. 162-181.

[4] M. Brstle et al., Sosse - Simple Operating System for Smartcard Education, www.mbsks.franken.de/sosse/index.html.

[5] G. Canivet, Analyse des effets d'attaques par fautes et conception sécurisée sur plate-forme reconfigurable, Ph.D. thesis, Institut polytechnique de Grenoble, 2009.

[6] F. Darracq, T. Beauchêne, V. Pouget, H. Lapuyade, D. Lewis, P. Fouillat and A. Touboul, Single-event sensitivity of a single SRAM cell, IEEE Transactions on Nuclear Science, vol. 49 (3), IEEE, 2002, pp. 1486 1490.

[7] P. Dusart, G. Letourneux and O. Vivolo, Differential fault analysis on A.E.S., Proceedings of the Int. Conf. on Applied Cryptography and Network Security - ACNS 2003, LNCS, vol. 2846, Springer-Verlag, 2003, pp. 293-306.

[8] Ch. Giraud, DFA on AES, Proceedings of AES 2004, LNCS, vol. 3373, Springer-Verlag, 2005, pp. 27-41.

[9] A. Moradi, M.T. Manzuri Shalmani and M. Salmasizadeh, A generalized method of differential fault attack against AES cryptosystem, Cryptographic Hardware and Embedded Systems - Proceedings of CHES 2006, LNCS, vol. 4249, Springer-Verlag, 2006, pp. 91-100.

[10] National Institute of Standards and Technology (NIST), Announcing the advanced encryption standard (AES), Federal Information Processing Standards Publication, vol. 197, 2001.

[11] G. Piret and J.J. Quisquater, A differential fault attack technique against SPN structure with application to the AES and KHAZAD, Cryptographic Hardware and Embedded Systems - Proceedings of CHES 2003, LNCS, vol. 2779, Springer-Verlag, 2003, pp. 77-88.

[12] V. Pouget, Test et analyse par faisceau laser: Plateforme et applications, Journée thématique du GDR SOC-SIP, 2007. www.lirmm.fr/soc_sip/ 6fev/GCT_R1_Pouget.pdf

[13] S. P. Skorobogatov and R. J. Anderson, Optical fault induction attacks, Cryptographic Hardware and Embedded Systems - Proceedings of CHES 2002, LNCS, vol. 2523, Springer-Verlag, 2002, pp. 2-12.

[14] A. Tria, B. Robisson, J.M. Dutertre and A.P. Mirbaha, Fault attacks from theory to practise: what is possible to do?, 2-nd CanadaFrance Workshop on Foundations \& Practice of Security, 2009. www-mitacs2009.imag.fr/Material/mitac_part1.pdf and mitac_part2. pdf 